\title{
Turbulence et structure
}

\author{
Raoul Robert*
}

\begin{abstract}
Lorsqu'on observe notre environnement à grande échelle, on voit de la structure. Penser notamment aux grands tourbillons cycloniques ou anticycloniques qui ont un diamètre de l'ordre du millier de kilomètres et qui parcourent l'atmosphère terrestre. Des structures semblables existent dans les atmosphères des planètes gazeuses en rotation rapide comme Jupiter ou Saturne. Une structure spectaculaire comme la grande tache rouge de Jupiter est un gigantesque tourbillon (environ le diamètre de la terre) qui intéresse une mince pellicule à la surface de la planète, ce phénomène est d'une remarquable stabilité, il perdure depuis au moins trois siècles puisqu'il a été observé pour la première fois par Galilée. Plus loin dans l'univers nous pouvons observer parmi les Galaxies un petit nombre de formes caractéristiques (Galaxies sphériques, elliptiques, barrées, spirales, spirales barrées). Le caractère commun remarquable de ces structures est qu'elles se forment et perdurent au sein d'un fluide fortement turbulent (un gas d'étoiles dans le cas des galaxies). La compréhension physique de ces beaux phénomènes a occupé pas mal de monde depuis le début du siècle et soulevé nombre de questions fondamentales.
\end{abstract}

\section{Des tourbillons atmosphériques à la formation des galaxies}

Dans le cas des tourbillons atmosphériques (ou structures cohérentes) c'est Onsager [17] qui le premier (en 1949) comprit que c'était un phénomène typique de la dimension deux et qu'il devait avoir une explication en terme de mécanique statistique de l'équation d'Euler du fluide parfait bidimensionnel.

Dans le cas des Galaxies la première idée naturelle consiste à faire de façon classique (ce qui au demeurant ne va pas sans quelques difficultés dues à la nature singulière du potentiel gravitationnel, voir Padmanabhan [18]) la mécanique statistique d'un nuage d'étoiles. On tombe alors sur quatre problèmes sérieux.

1. La théorie prédit qu'on doit avoir une ségrégation des masses des étoiles, les plus massives devant se regrouper au centre. Ce phénomène n'est pas observé.

2. Le système n'a pas d'état d'équilibre dans tout l'espace: il peut s'étaler indéfiniment tout en augmentant son entropie et conservant la valeur des invariants énergie, impulsion et moment angulaire.

3. En supposant qu'on confine artificiellement le système dans une boule de l'espace physique il n'y a toujours pas de maximum d'entropie pour une énergie et un moment angulaire fixés (une partie de la matière peut se concentrer au centre où la densité devient infinie et l'entropie tend vers l'infini: c'est la catastrophe gravotherme mise en évidence par Antonov en 1962 [2] et qui pourrait être au point de départ de la formation des trous noirs).

${ }^{*}$ CNRS UMR 5585, Université Lyon 1. 
4. Pour le système confiné, il peut néammoins exister des maxima locaux de l'entropie. Un calcul fait par Chandrasekhar [4] permet d'estimer le temps de relaxation du système stellaire vers un tel équilibre sous l'effet des collisions entre étoiles (on appelle ici collision le passage de deux étoiles à proximité l'une de l'autre; la perturbation de vitesse qui en découle génère un processus de diffusion). Chandrasekhar a trouvé pour ce temps de relaxation collisionnel:

$$
t_{\text {coll }}=\frac{N}{\log N} t_{d}
$$

où $N$ est le nombre d'étoiles de la galaxie et $t_{d}$ le temps dynamique, c'est à dire le temps moyen mis par une étoile pour faire un tour de la galaxie (pour la voie lactée $N \approx 10^{11}$ et $t_{d} \approx 10^{8}$ ans, d'où $t_{\text {coll }} \approx 10^{10} t_{d}$ ). Sachant que l'âge de l'univers est d'environ $100 t_{d}$, on voit qu'un tel mécanisme est beaucoup trop lent pour expliquer quoi que ce soit.

D'où l'idée émise par Hénon [8], King [10] et Lynden-Bell [12] que ce n'est pas le processus de collision (elles sont beaucoup trop rares) qui conduit le système vers une sorte d'équilibre mais un processus de mélange beaucoup plus rapide qui apparaît dans l'équation de Vlasov-Poisson (sans collisions) qui régit l'évolution de la densité dans l'espace des phases. Ils ont appelé ce mécanisme la relaxation rapide.

Il y a une croyance assez populaire selon laquelle les galaxies spirales seraient des espèces de tourbillons (à cause de leur aspect bidimensionnel et de leur rotation clairement visible), mais cette analogie est trompeuse, la véritable analogie réside dans la structure mathématique similaire des équations d'Euler bidimensionnelles et des équations de Vlasov-Poisson, qui peuvent se prêter à un traitement de mécanique statistique identique.

Nous explicitons au paragraphe suivant la similarité entre les équations d'Euler $2 d$ incompressibles et les équations de Vlasov-Poisson.

\section{Les équations d'Euler 2D et Vlasov-Poisson}

\section{1 Équations d'Euler}

Les équations d'Euler qui régissent le mouvement d'un fluide parfait incompressible occupant un domaine borné $\Omega$ du plan s'écrivent, sous la forme vitesse-tourbillon:

$$
\left\{\begin{array}{l}
\omega_{t}+\operatorname{div}(\omega \mathbf{u})=0, \\
\operatorname{rot} \mathbf{u}=\omega, \operatorname{divu}=0, \quad \mathbf{u} \cdot \mathbf{n}=0 \text { sur } \partial \Omega
\end{array}\right.
$$

où $\mathbf{u}(t, \mathbf{x})$ est le champ de vitesse du fluide, $\omega=$ rotu (scalaire) la fonction tourbillon ou vorticité, $\mathbf{n}$ la normale unité sortante sur $\partial \Omega$. La condition d'incompressibilité se traduit par l'existence d'une fonction de courant $\psi(t, \mathbf{x})$ :

$$
\omega=-\Delta \psi, \quad \psi=0 \text { sur } \partial \Omega \text {. }
$$

Les invariants de ce système dynamique sont:

- L’énergie cinétique

$$
\Xi(\omega)=\frac{1}{2} \int_{\Omega} \mathbf{u}^{2} d \mathbf{x}=\frac{1}{2} \int_{\Omega} \psi \omega d \mathbf{x}
$$

- Toutes les fonctionnelles

$$
F_{\Theta}(\omega)=\int_{\Omega} \Theta(\omega(\mathbf{x})) d \mathbf{x}
$$

ESAIM: Proc., VOL. 3, 1998, 119-129 
pour n'importe quelle fonction continue $\Theta$.

Quantités auxquelles il faut rajouter:

- le moment angulaire par rapport à 0 si $\Omega$ est un disque centré en $0, \Omega=B(0, R)$ :

$$
\mathbf{M}(\omega)=\int_{\Omega} \mathbf{x} \wedge \mathbf{u}(\mathbf{x}) d \mathbf{x}=\frac{1}{2}\left(\int_{\Omega}\left(R^{2}-\mathbf{x}^{2}\right) \omega(\mathbf{x}) d \mathbf{x}\right) e_{3} .
$$

$-\int_{\Omega} \mathbf{x} \omega(\mathbf{x}) d \mathbf{x}$, si $\Omega=\mathbb{R}^{2}$

\section{2 Équations de Vlasov-Poisson}

Soit $f(t, \mathbf{x}, \mathbf{v})$ une fonction scalaire positive décrivant la densité de répartition des étoiles d'une galaxie (dans l'espace des phases $(\mathbf{x}=$ position, $\mathbf{v}=$ vitesse)). Si l'on néglige l'effet des collisions (i.e. deux étoiles passant à proximité l'une de l'autre), cette densité doit vérifier l'équation de Vlasov-Poisson:

$$
\frac{\partial f}{\partial t}+\operatorname{div}_{x}(\mathbf{v} f)+\operatorname{div}_{\mathbf{v}}(\mathbf{E} f)=0,
$$

où $\mathbf{E}(t, \mathbf{x})$ est le champ gravitationnel, $\mathbf{E}=-\nabla_{x} \Phi, \Phi(t, \mathbf{x})=-G \int \frac{\rho\left(t, \mathbf{x}^{\prime}\right)}{\left|\mathbf{x}-\mathbf{x}^{\prime}\right|} d \mathbf{x}^{\prime}$ est le potentiel gravitationnel et $\rho(t, \mathbf{x})=\int f(t, \mathbf{x}, \mathbf{v}) d \mathbf{v}$ la densité spatiale d'étoiles.

Les quantités conservées par (2) sont:

- L'énergie totale (cinétique + potentielle):

$$
\Xi(f)=\iint \frac{1}{2} \mathbf{v}^{2} f(\mathbf{x}, \mathbf{v}) d \mathbf{x} d \mathbf{v}+\frac{1}{2} \int \rho \Phi d \mathbf{x}
$$

- Les intégrales

$$
\iint \Theta(f) d \mathbf{x} d \mathbf{v}
$$

pour toute fonction continue $\Theta$ telle que $\Theta(0)=0$.

- L’impulsion

$$
\iint \mathbf{v} f d \mathbf{x} d \mathbf{v}
$$

- Le moment angulaire

$$
\iint \mathbf{x} \wedge \mathbf{v} f d \mathbf{x} d \mathbf{v}
$$

En notant $\mathbf{U}=\left(\begin{array}{l}v \\ \mathrm{E}\end{array}\right)$ le champ de vecteur sur $\mathbb{R}^{6}$, on remarque que $\operatorname{div}_{6}(\mathbf{U})=0$ et que (2) peut s'écrire:

$$
f_{t}+\operatorname{div}_{6}(f \mathbf{U})=0,
$$

de sorte que les équations (1) et (2) se mettent sous une forme commune

$$
\left\{\begin{array}{l}
q_{t}+\operatorname{div}(q \mathbf{U})=0 \\
\mathbf{U}=\mathcal{L}(q)
\end{array}\right.
$$

où $q(t, \mathbf{x})$ est une fonction scalaire et $\mathbf{U}(t, \mathbf{x})$ un champ de vitesse incompressible qui est déterminé à partir de $q$ au moyen d'un opérateur intégro-différentiel $\mathcal{L}$. 


\subsection{Résolution du problème de Cauchy}

Il n'existe malheureusement pas actuellement de théorème d'existence-unicité général, pour le problème de Cauchy, pour des équations du type (3). Aussi nous allons examiner séparément les cas de (1) et (2).

Pour l'équation d'Euler la situation est satisfaisante depuis le théorème de Youdovitch [29] (1963) qui dit que pour toute donnée initiale $\omega_{0}(\mathbf{x})$ dans $L^{\infty}(\Omega)$, il existe une solution faible unique de $(1)$. La solution faible $\omega(t, \mathbf{x})$ est dans $L^{\infty}(\Omega)$ pour tout $t$ et appartient à l'espace $C\left(\left[0, \infty\left[; L^{p}(\Omega)\right)\right.\right.$ pour tout $p, 1 \leq p<\infty$.

On notera $\omega(t, \cdot)=\mathcal{F}_{t} \omega_{0}, \mathcal{F}_{t}$ désignant le flot de l'équation d'Euler sur l'espace des phases $L^{\infty}(\Omega)$.

En ce qui concerne l'équation de Vlasov-Poisson, la situation ne s'est éclaircie que plus récemment.

On trouvera par exemple un résultat d'existence d'une solution régulière (pour une donnée initiale $f_{0}(\mathbf{x}, \mathbf{v})$ régulière) dans Bardos et Degond [3].

Pour $f_{0}$ dans $L^{1}\left(\mathbb{R}^{6}\right) \cap L^{\infty}\left(\mathbb{R}^{6}\right)$, vérifiant $\iint \frac{1}{2} \mathbf{v}^{2} f_{0} d \mathbf{x} d \mathbf{v}<\infty$, on a existence de solutions faibles (Horst et Hunze 1984 [9]).

L'unicité est plus délicate à obtenir, il faut rajouter des hypothèses sur la condition initiale, citons deux résultats récents:

- avec une condition de Lipschitz sur $f_{0}$ (Lions et Perthame 1991 [11])

- en supposant $f_{0} L^{\infty}$ à support compact (Robert 1997 [21]).

Finalement, pour ce qui nous intéresse ici, lorsque $f_{0}$ est $L^{\infty}$ à support compact on a exactement l'analogue du théorème de Youdovitch, avec une solution faible unique $f(t, \mathbf{x}, \mathbf{v})$ dans $C\left(\left[0, \infty\left[; L^{p}\left(\mathbb{R}^{6}\right)\right)\right.\right.$ pour tout $p, 1 \leq p<\infty$, qui définit un flot $\mathcal{F}_{t}$ sur $L^{\infty}\left(\mathbb{R}^{6}\right)$.

Grâce aux estimations sur les moments de [11], on peut montrer qu'une telle solution faible reste à support compact pour tout temps.

\section{Mécanique statistique de la relaxation rapide}

Pour un système dynamique tel que (1) ou (2) on a vu que la fonction scalaire qui définit l'état du système (vorticité ou densité) est convectée par un champ de vitesse incompressible. En général, cette fonction va développer des oscillations au voisinage de tout point. C'est ce comportement que nous visualisons comme un désordre turbulent.

Le but de l'entreprise est d'expliciter une fonctionnelle entropie qui mesure précisément cette notion vague de désordre turbulent au sein du fluide. Cette fonctionnelle est la même dans le cas des équations d'Euler ou de Vlasov. La méthode consiste tout d'abord à construire une famille d'approximations de ces équations, qui possèdent des mesures de Liouville invariantes par le flot approché. On montre ensuite une propriété de grandes déviations de ces mesures lorsqu'on les plonge dans l'espace des mesures d'Young, qui est ici l'espace naturel dans lequel prendre la limite thermodynamique. La fonctionnelle qui sert à mesurer les grandes déviations est l'entropie cherchée.

\section{Une famille d'approximations.}

Considérons tout d'abord le cas de l'équation d'Euler. Soit $h$ un paramètre $>0$, nous notons $L_{h}(\Omega)$ le sous espace des fonctions de $L^{2}(\Omega)$, à support compact dans $\Omega$ et constantes presque partout sur les cubes $\left.h\left(Q_{2}+j\right), Q_{2}=\right]-1 / 2,1 / 2\left[{ }^{2}, j \in \mathbb{Z}^{2} . L_{h}(\Omega)\right.$ est un espace de Hilbert de dimension finie, il est donc naturellement muni d'une mesure de volume.

\section{Théorème d'approximation.}


Il existe un système dynamique, $\mathcal{F}_{t}^{h}$ sur $L_{h}(\Omega)$, qui conserve la mesure de volume et possède la propriété suivante:

Pour tout $\omega_{0}$ donné dans $L^{\infty}(\Omega)$, pour tout $T>0$ et pour tout $\varepsilon>0$, il existe $h_{0}>0$ tel que pour tout $h \leq h_{0}$ existe $\omega_{0 h}$ dans $L_{h}(\Omega)$ tel que

$$
\left\|\mathcal{F}_{t}^{h} \omega_{0 h}-\mathcal{F}_{t} \omega_{0}\right\|_{L^{2}(\Omega)} \leq \varepsilon \operatorname{sur}[0, T] .
$$

On a un résultat tout à fait analogue pour l'équation de Vlasov-Poisson:

Soit $L_{h}\left(Q_{6}\right)$ le sous espace des fonctions de $L^{2}\left(Q_{6}\right)$ qui sont constantes sur les cubes $h Q_{6}+h j($ ici $h=1 / N)$.

Il existe un système dynamique $\mathcal{F}_{t}^{h}$ sur $L_{h}\left(Q_{6}\right)$, qui préserve la mesure de volume et vérifiant:

pour toute solution faible (de donnée initiale $f_{0}$ bornée) restant à support compact dans $Q_{6}$ sur l'intervalle $[0, T]$ (on peut toujours s'y ramener par un changement d'échelle convenable en espace et en temps) et pour tout $\varepsilon>0$, il existe $h_{0}>0$ tel que pour tout $h \leq h_{0}$ il existe $f_{0 h}$ dans $L_{h}\left(Q_{6}\right)$ tel que

$$
\left\|\mathcal{F}_{t}^{h} f_{0 h}-\mathcal{F}_{t} f_{0}\right\|_{L^{2}\left(Q_{6}\right)} \leq \varepsilon \operatorname{sur}[0, T]
$$

\section{Limites à long terme et mesures d'Young.}

Considérons, pour fixer les idées, l'équation (1) avec une condition initiale $\omega_{0}$. Lorsque $t$ croit, $\mathcal{F}_{t} \omega_{0}$ va devenir une fonction oscillante. Soir $r=\left\|\omega_{0}\right\|_{L^{\infty}(\Omega)}$; puisque la vorticité est convectée par le champ de vitesse, $\mathcal{F}_{t} \omega_{0}$ va rester dans la boule $L_{r}^{\infty}=\left\{\infty:\|\omega\|_{\infty} \leq\right.$ $r\}$. On peut toujours supposer (au besoin en extrayant une sous suite) que $\mathcal{F}_{t} \omega_{0}$ converge faiblement (pour la topologie faible étoile $\sigma\left(L^{\infty}, L^{1}\right)$ ) vers une fonction $\omega^{*}$. On voit alors facilement que $F_{\Theta}\left(\mathcal{F}_{t} \omega_{0}\right)$ ne converge pas vers $F_{\Theta}\left(\omega^{*}\right)$ si $\Theta$ n'est pas linéaire. Ainsi beaucoup d'information (donnée par les invariants) est perdue dans cette limite, et l'espace $L^{\infty}$ faible étoile n'est pas le bon candidat pour décrire les limites à long terme. Fort heureusement le bon espace pour faire ça est bien connu. L'intérêt de décrire d'une façon macroscopique les oscillations à petite échelle des fonctions fut compris il y a longtemps par Young [30]. Pour résoudre des problèmes de calcul des variations, Young introduisit une généralisation naturelle de la notion de fonction: à tout point $\mathbf{x}$ de $\Omega$ on n'associe plus une valeur réelle bien déterminée mais seulement une mesure de probabilité sur $\mathbb{R}$ (une telle application est appelée une mesure d'Young sur $\Omega \times \mathbb{R}$ ). Plus précisément, une mesure d'Young $\nu$ sur $\Omega \times \mathbb{R}$ est une application mesurable $\mathbf{x} \rightarrow \nu_{x}$ de $\Omega$ dans l'ensemble $\mathbf{M}_{1}(\mathbb{R})$ des probabilités Boreliennes sur $\mathbb{R}$, muni de la topologie étroite (topologie faible associée aux fonctions continues bornées). Il est clair qu'une telle application $\nu$ définit une mesure de Borel positive sur $\Omega \times \mathbb{R}$ (que l'on notera toujours $\nu$ ) par:

$$
\langle\nu, \varphi\rangle=\int_{\Omega}\left\langle\nu_{x}, \varphi(\mathbf{x}, \cdot)\right\rangle d \mathbf{x}
$$

pour toute fonction $\varphi(\mathbf{x}, z)$, continue à support compact sur $\Omega \times \mathbb{R}$.

A toute fonction réelle mesurable $g$ sur $\Omega$, on associe la mesure d'Young $\delta_{g}: \mathbf{x} \rightarrow \delta_{g(\mathbf{x})}$, masse de Dirac en $g(\mathbf{x})$. On désignera par $\mathcal{M}$ le convexe des mesures d'Young sur $\Omega \times \mathbb{R}$, rappelons quelques propriétés utiles.

- $\mathcal{M}$ est fermé dans l'espace des mesures Boreliennes bornées sur $\Omega \times \mathbb{R}$ (muni de la topologie étroite). Pour la suite $\mathcal{M}$ sera muni de cette topologie étroite. Si on remplace $\mathbb{R}$ par l'intervalle compact $[-r, r]$, l'espace $\mathcal{M}_{r}$ des mesures d'Young sur $\Omega \times[-r, r]$ est compact. 
- $\left\{\delta_{g} \mid g: \Omega \rightarrow[-r, r]\right.$, mesurable $\}$ est dense dans $\mathcal{M}_{r}$.

On peut maintenant identifier les limites à long terme avec des mesures d'Young. En effet, $\mathcal{M}_{r}$ est une compactification convenable de $L_{r}^{\infty}$ car la convergence étroite (lorsque $t$ tend vers l'infini) de $\delta_{\mathcal{F}_{t} \omega_{0}}$ vers une mesure d'Young $\nu$ conserve l'information donnée par les invariants; en effet, pour toute fonction continue $\varphi(z)$ :

$$
\int_{\Omega} \varphi\left(\mathcal{F}_{t} \omega_{0}\right) d \mathbf{x} \rightarrow \int_{\Omega}\left\langle\nu_{\mathbf{x}}, \varphi\right\rangle d \mathbf{x}
$$

et comme l'expression de gauche est constante et égale à $\left\langle\pi_{\omega 0}, \varphi\right\rangle$, où $\pi_{\omega 0}$ est la mesure image de $d \mathbf{x}$ par $\omega_{0}$, il vient:

$$
\int_{\Omega} \nu_{\mathbf{x}} d \mathbf{x}=\pi_{\omega 0}
$$

De même pour l'énergie, $\mathcal{F}_{t} \omega_{0}$ converge faiblement vers $\bar{\nu}(x)=\int z d \nu_{x}(z)$, d'où $\Xi\left(\mathcal{F}_{t} \omega_{0}\right)$ $\rightarrow \Xi(\bar{\nu})$, qui est l'énergie de la mesure d'Young $\nu$, et donc:

$$
\Xi(\bar{\nu})=\Xi\left(\omega_{0}\right) \text {. }
$$

On voit donc que les invariants imposent des contraintes sur les limites à long terme éventuelles.

\section{Les états d'équilibre statistique.}

Considérons à nouveau le cas de l'équation d'Euler.

L'espace $L_{h}(\Omega)$ est formé des fonctions tourbillon de la forme $\omega_{h}(\mathbf{x})=\sum_{j} \omega_{h}^{j} \chi\left(\frac{x}{h}-j\right)$, pour $j$ dans un certain ensemble fini ( $\chi$ désigne la fonction caractéristique de $Q_{2}$ ). On va

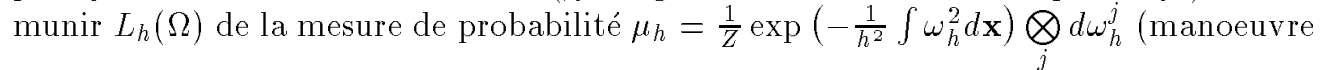
autorisée puisque $\int \omega^{2} d \mathbf{x}$ est invariant par le flot de l'équation d'Euler).

Remarquer par $\mu_{h}$ s'écrit aussi $\mu_{h}=\bigotimes_{j} d \pi_{*}\left(\omega_{h}^{j}\right)$, où $d \pi_{*}(\omega)=\frac{1}{\sqrt{\pi}} \exp \left(-\omega^{2}\right) d \omega$.

On peut donc considérer $\omega_{h}$ comme une fonction tourbillon aléatoire suivant la loi $\mu_{h}$. $\mathrm{Si}$ nous considérons maintenant la famille (dépendant de $h$ ) des mesures d'Young aléatoires $\delta_{\omega h}$ sur $\Omega \times \mathbb{R}$, il découle d'un théorème général [14] qu'elle possède la propriété des grandes déviations avec $1 / h^{2}$ et la fonctionnelle information $I_{\pi^{*}}(v)$. Ce qui signifie (en gros) que pour tout Borelien $B$ de $\mathcal{M}$, on a:

$$
\operatorname{Prob}\left(\delta_{\omega_{h}} \in B\right) \sim \exp \left(-\frac{1}{h^{2}} \inf _{\nu \in B} I_{\pi^{*}}(\nu)\right), \text { quand } h \rightarrow 0 ;
$$

où $\pi^{*}=d x \otimes \pi_{*}$ et $I_{\pi^{*}}(\nu)$ est la fonctionnelle information de Kullback classique définie sur $\mathcal{M}$ par:

$$
I_{\pi^{*}}(\nu)=\int \log \frac{d \nu}{d \pi^{*}} d \nu
$$

si $\nu$ est absolument continue par rapport à $\pi^{*}$,

$$
I_{\pi^{*}}(\nu)=+\infty \quad \text { sinon }
$$

La conséquence de cette propriété est que les mesures d'Young aléatoires $\delta_{w h}$ conditionnées par les contraintes (4), (5) se concentrent exponentiellement autour de l'ensemble $\mathcal{E}^{*}$ des solutions du problème variationnel

$$
I_{\pi^{*}}\left(\nu^{*}\right)=\inf \left\{I_{\pi^{*}}(\nu) \mid \nu \in \mathcal{E}\right\}
$$


où $\mathcal{E}$ est le sous ensemble fermé des mesures d'Young vérifiant les contraintes (4) et (5) (voir [14]).

Notons maintenant $\pi_{0}=\frac{1}{\Omega} \pi_{\omega 0}$, et $\pi=d \mathbf{x} \otimes \pi_{0}$, pour tout $\nu$ dans $\mathcal{E}$, on vérifie la formule: $I_{\pi^{*}}(\nu)=I_{\pi}(\nu)+|\Omega| I_{\pi_{*}}\left(\pi_{0}\right)$. Donc si $I_{\pi_{*}}\left(\pi_{0}\right)<\infty$, il revient au même de résoudre le problème variationnel avec la fonctionnelle $I_{\pi}(\nu)$. C'est ce que nous ferons dans la suite; pour la justification dans le cas où $\pi_{0}$ n'est pas absolument continue par rapport à $\pi_{*}$ voir [14, 22].

C'est donc en définitive le problème variationnel suivant qui définit l'ensemble $\mathcal{E}^{*}$ des états d'équi

libre correspondant à une certaine donnée initiale $\omega_{0}$.

$$
I_{\pi}\left(\nu^{*}\right) \inf \left\{I_{\pi}(\nu) \mid \nu \in \mathcal{E}\right\}
$$

L'équation de Vlasov-Poisson se traite de façon tout à fait analogue, il suffit de remplacer $\Omega$ par $Q_{6}$ et on se ramène au problème variationnel (6), où cette fois $\pi=d \mathbf{x} \otimes \pi_{f_{0}}$ et $\mathcal{E}$ désigne l'ensemble des mesures d'Young sur $Q_{6} \times \mathbb{R}$ qui vérifient les contraintes

$$
\frac{1}{2} \iint_{Q_{6}} \mathbf{v}^{2} \bar{\nu}(\mathbf{x}, \mathbf{v}) d \mathbf{x} d \mathbf{v}+\frac{1}{2} \int_{Q_{3}} \bar{\rho} \bar{\Phi} d \mathbf{x}=\Xi\left(f_{0}\right) \text {, énergie }
$$

où $\bar{\rho}(\mathbf{x})=\int_{Q_{3}} \bar{\nu}(\mathbf{x}, \mathbf{v}) d \mathbf{v}$ et $\bar{\Phi}$ est le potentiel gravitationnel associé à $\bar{\rho}$ (étendu par 0 en dehors de $Q_{3}$ ).

$$
\begin{gathered}
\iint_{Q_{6}} \nu_{x, v} d \mathbf{x} d \mathbf{v}=\pi_{f_{0}} \\
\iint_{Q_{6}} \mathbf{v} \bar{\nu}(\mathbf{x}, \mathbf{v}) d \mathbf{x} d \mathbf{v}=\mathbf{P}_{0}, \text { impulsion } \\
\iint_{Q_{6}} \mathbf{x} \wedge \mathbf{v} \bar{\nu}(\mathbf{x}, \mathbf{v}) d \mathbf{x} d \mathbf{v}=\mathbf{M}_{0}, \text { moment angulaire. }
\end{gathered}
$$

En résumé nous avons ramené le problème de la détermination des états d'équilibre à la résolution d'un problème variationnel (6) que nous allons examiner au paragraphe suivant.

\section{Détermination des états d'équilibre}

\section{Equation d'Euler.}

Considérons le problème variationnel (6) écrit au paragraphe précédent. Comme il est assez facile de voir que la fonctionnelle $I_{\pi}(\nu)$ est inf-compacte sur $\mathcal{M}$ muni de la topologie étroite et que $\xi$ est un ensemble fermé non vide, il existe toujours au moins une solution.

Nous allons maintenant expliciter un peu plus les choses dans un cas simple. Pour cela supposons que la vorticité initiale $\omega_{0}$ prenne seulement $n$ valeurs distinctes $a_{1}, \ldots, a_{n}$, sur les ensembles $\Omega^{1}, \ldots, \Omega^{n}$. Il est alors facile de voir que les mesures d'Young $\nu$ qui vérifient la contrainte (4) s'écrivent: $\nu_{\mathbf{x}}=\sum p_{i}(\mathbf{x}) \delta a_{j}$, où $p_{i}(\mathbf{x}) \geq 0, \sum p_{i}(\mathbf{x})=1$; la fonction mesurable $p_{i}(\mathbf{x})$ donne la probabilité de trouver la valeur $a_{i}$ au point $\mathbf{x}$.

(6) se ramène alors à minimiser

$$
\int_{\Omega} \sum p_{i} \log p_{i} d \mathbf{x}
$$


sous les contraintes

$$
\begin{gathered}
\sum p_{i}(\mathbf{x})=1, \text { pour tout } \mathbf{x}, \\
\int_{\Omega} p_{i}(\mathbf{x}) d \mathbf{x}=\left|\Omega^{i}\right|, \text { pour } i=1, \ldots, n, \\
\Xi\left(\sum a_{i} p_{i}\right)=\Xi\left(\omega_{0}\right) .
\end{gathered}
$$

Soit $\left(p_{1}^{*}, \ldots, p_{n}^{*}\right)$ une solution de (6) telle que les fonctions $p_{i}^{*}$ soient continues et strictement positives sur $\Omega$. On peut alors montrer qu'il existe des multiplicateurs de Lagrange $\alpha_{1}, \ldots, \alpha_{n}$, $\beta$ (associés aux contraintes (8), (9)), tels que

$$
p_{i}^{*}(\mathbf{x})=\frac{1}{Z\left(\psi^{*}(\mathbf{x})\right)} \exp \left(-\alpha_{i}-\beta a_{i} \psi^{*}(\mathbf{x})\right), \quad i=1, \ldots, n,
$$

où $Z(\psi)=\sum_{i} \exp \left(-\alpha_{i}-\beta a_{i} \psi\right)$, et $\psi^{*}$ est la fonction de courant associée à $\omega^{*}=\sum a_{i} p_{i}^{*}$. Et donc $\psi^{*}$ vérifie l'équation des "états de Gibbs":

$$
\left\{\begin{array}{l}
-\Delta \psi^{*}=\frac{\sum a_{i} \exp \left(-\alpha_{i}-\beta a_{i} \psi^{*}\right)}{Z\left(\psi^{*}\right)}, \\
\psi^{*}=0 \text { sur } \partial \Omega
\end{array}\right.
$$

Cette équation élliptique non linéaire, dont le second membre est une fonction continue bornée de $\psi^{*}$, a des solutions pour toutes les valeurs des paramètres. La solution est unique pour $\beta$ plus grand qu'une certaine valeur critique strictement négative $\beta_{c}$, mais lorsque $-\beta$ devient grand, on peur avoir des bifurcations et apparition de solutions multiples [27].

Remarque.

Les états d'équilibre observés physiquement correspondent en fait aux vorticités moyennes $\omega^{*}=\sum a_{i} p_{i}^{*}$.

Equation de Vlasov-Poisson.

Le problème (6) décrit au paragraphe précédent a lui aussi toujours des solutions, pour les mêmes raisons de compacité. Nous renvoyons à $[5,6,22]$ pour une discussion détaillée de ces solutions. Signalons cependant une différence essentielle avec le cas précédent, c'est qu'ici, à long terme, le système n'a aucune raison de rester confiné. Et donc il n'est pas physiquement pertinent de résoudre le problème variationnel dans un borné de $\mathbb{R}^{6}$. Si on essaye alors de résoudre le problème variationnel, sans aucun confinement, dans tout $\mathbb{R}^{6}$, on trouve qu'il n'y a pas de solution, le système pouvant s'étaler indéfiniment tout en augmentant son entropie. Au terme d'une discussion physique assez complexe [5,6], on peut montrer que les états physiquement pertinents s'obtiennent en résolvant le problème variationnel sur $B \times \mathbb{R}^{3}$, où $B$ est une boule de l'espace physique. On peut montrer qu'alors la catastrophe gravotherme ne se produit plus et que ce problème à bien au moins une solution [22].

\section{Relaxation vers l'équilibre et modélisation des petites échelles}

Un des principaux intérêts d'une théorie de l'équilibre statistique est de permettre l'utilisation des recettes classiques de la thermodynamique linéaire au voisinage de l'équilibre pour construire de nouvelles équations décrivant le comportement macroscopique du système (c'est ainsi que l'on obtient la plupart des équations physiques macroscopiques). Le même programme peut être mis en oeuvre pour la relaxation rapide, le but étant d'obtenir des équations d'évolution qui décrivent le comportement des grandes échelles sans avoir à se préoccuper 
du détail des petites. Pour Euler et Vlasov-Poisson, de telles équations de "relaxation" peuvent être obtenues de manière simple au moyen d'un nouveau principe de thermodynamique hors équilibre d'une portée générale: le principe de maximum de production d'entropie, qui est discuté en [26, 23, 19]. On trouvera un exposé détaillé du cas Euler dans [23] et du cas Vlasov-Poisson dans [6]. Nous donnerons seulement un aperçu de ces équations de relaxation en considérant le cas le plus simple de l'équation d'Euler avec pour $\omega_{0}$ une tache de vorticité de niveau a entourée de vorticité nulle. Dans ce cas, en désignant par $\bar{\omega}$ la fonction vorticité localement moyennée à une certaine échelle spatiale, on trouve le système d'équations de relaxation suivant $[6,23]$ :

$$
\left\{\begin{array}{l}
\bar{\omega}_{t}+\operatorname{div}(\bar{\omega} \overline{\mathbf{u}}+\mathbf{J})=0, \\
\mathbf{J}=-c \bar{\omega}(a-\bar{\omega})[\nabla \bar{\omega}+\beta \bar{\omega}(a-\bar{\omega}) \nabla \bar{\psi}] \\
\mathbf{J} \cdot \mathbf{n}=0 \text { sur } \partial \Omega, \\
\beta=-\int_{\Omega} \nabla \bar{\psi} \cdot \nabla \bar{\omega} \bar{\omega}(a-\bar{\omega}) d \mathbf{x} / \int_{\Omega}(\nabla \bar{\psi})^{2} \bar{\omega}^{2}(a-\bar{\omega})^{2} d \mathbf{x} .
\end{array}\right.
$$

où $\bar{\psi}$ est la fonction de courant de $\bar{\omega}, \overline{\mathbf{u}}=\operatorname{rot} \bar{\psi}, c$ est une constante fixée empiriquement (en fonction de la taille des plus petites échelles que l'on désire observer), et $\beta$ est un scalaire ajusté à chaque instant de manière à conserver l'énergie.

On voit que (11) est une équation de convection-diffusion qui ressemble assez aux équations de Navier-Stokes, avec toutefois la différence importante qu'elle conserve l'énergie et la vorticité totale et conduit à long terme le système vers un état d'équilibre non trivial. L'expression donnant $\mathbf{J}$ s'interprète classiquement comme une relation de fermeture turbulente.

Ceci s'étend à une vorticité initiale $\omega_{0}$ quelconque [23].

La même méthode appliquée a l'équation de Vlasov-Poisson conduit à des équations de relaxation de type Fokker-Planck [6].

\section{Commentaires}

Les résultats d'approximation présentés dans la section 3 répondent à une question déjà abordée dans [14], on en trouvera une preuve dans [22].

La pertinence de la théorie de l'équilibre statistique pour l'équation d'Euler $2 d$ a été testée par des expériences de laboratoire ainsi que par des simulations numériques directes utilisant les équations de Navier-Stokes à grand nombre de Reynolds. Dans ces tests on considère des situations où un fluide turbulent converge vers un état d'équilibre et on compare la relation $\omega=f(\psi)$ obtenue expérimentalement avec la prédiction théorique [7, 27, 28].

Outre leur intérêt théorique, les équations de relaxation ouvrent des perspectives en tant qu'outil de simulation, en effet elles fournissent en quelque sorte une paramétrisation optimale des petites échelles (voir [23] pour une application à la turbulence $2 d$ ). L'exploitation des équations analogues pour les systèmes stellaires est en cours.

L'équation de relaxation écrite au paragraphe précédent fait apparaître un terme non local $\beta$ qui complique un peu l'étude mathématique du problème de Cauchy [15], mais ceci n'engendre pas de réelle difficulté numérique [23].

Le recours à la mécanique statistique permet donc d'obtenir de nouveaux modèles d'écoulements fluides, décrivant de façon efficace le mouvement à grande échelle sans avoir à se préoccuper explicitement des petites. Ceci est du au fait que le "chaos" à petite échelle engendré par la dynamique complexe de l'équation d'Euler (où de Vlasov) est bien pris en compte par la mécanique statistique. Pourquoi? Ceci est certainement lié à une propriété d'ergodicité du flot. Cette question à été clairement évoquée par Arnold [1], qui a montré que le mouvement du fluide parfait "ressemble" à un flot géodésique sur une variété Riemannienne à courbure sectionnelle négative (pour beaucoup de sections). Cette propriété qui implique 
l'instabilité exponentielle du flot n'est rien d'autre que le célèbre “effet papillon". C'est en fait ce mouvement chaotique et imprédictible à petite échelle qui rend l'organisation à grande échelle prédictible grâce á l'intervention de la mécanique statistique. Pour une discussion sur cet aspect des choses voir [24].

\section{References}

[1] V.I. ARnold, Ann. Inst. Fourier 16, (1966), 319-361.

[2] V.A. Antonov, Vest. Leningrad gos. Univ. 7, (1962), 135.

[3] C. Bardos \& P. Degond, Ann I.H.P., Anal. Nonlin., 2, (1985), 101-118.

[4] S. Chandrasekhar, Principles of stellar dynamics, Dover, 1942.

[5] P.H. Chavanis, Thèse, Ecole Normale Supérieure de Lyon, 1996.

[6] P.H. Chavanis, J. Sommeria \& R. Robert, Statistical mechanics of two-dimensional vortices and collisionless stellar systems. The Astrophysical Journal, 471, (1996), 385-399.

[7] M.A. Denoix, J. Sommeria \& A. Thess, Two-dimensional turbulence : the prediction of coherent structures by statistical mechanics. Proceedings of the 7 th Beer-Sheva Seminar on M.H.D. Flows and Turbulence, Jerusalem, 1993.

[8] M. Henon, An. Astrophys. 27, (1964), 83.

[9] E. Horst \& R. Hunze, Weak solutions of the initial value problem for the unmodified nonlinear Vlasov equation, Math. Meth. in the Appl. Sc. 6, (1984), 262.

[10] I.R. KING, Astrophysical J., 71, (1996), 64.

[11] P.L. Lions \& B. Perthame, Propagation of moments and regularity for the 3-dimensional Vlasov-Poisson system. Invent. Math. 105, (1991), 415-430.

[12] D. Lynden-Bell, Statistical mechanics of violent relaxation in stellar systems, Mon. Not. R. Astr. Soc. 181, (1967), 405.

[13] J. Michel \& R. Robert, Statistical mechanical theory of the great red spot of Jupiter, J. Stat. Phys. 77, 3/4, (1994), 645-666.

[14] J. Michel \& R. Robert, Large deviations for Young measures and statistical mechanics of infinite dimensional dynamical systems with conservation law, Comm. Math. Phys. 159, (1994), 195-215.

[15] A. Mikelic \& R. Robert, On the equations describing a relaxation towards a statistical equilibrium state in the two-dimensional perfect fluid dynamics, (to appear in SIAM J. of Math. Anal.).

[16] J. Miller, P.B. Weichman \& M.C. Cross, Statistical mechanics, Euler equations and Jupiter's red spot, Phys. Rev. A 45, (1992), 2328-2359.

[17] L. Onsager, Statistical hydrodynamics, Nuovo Cimento supll. 6, (1949), 279.

[18] T. Padmanabhan, Statistical mechanics of gravitating systems, Physics Reports, 188, $n^{\circ} 5,(1990), 285-362$.

[19] R. RoBERT, Relaxation towards a statistical equilibrium state in two-dimensional perfect fluid dynamics, Proceedings XIth International Congress of Mathematical Physics, Paris, 1994.

[20] R. Robert, A maximum entropy principle for two-dimensional Euler equations, J. Stat. Phys. 65, 3/4, (1991), 531-553.

[21] R. Robert, Unicité de la solution faible à support compact de l'équation de VlasovPoisson. C.R. Acad. Sci. Paris, 324, Série I, (1997), 873-877. 
[22] R. Robert, On the statistical mechanics of Euler and Vlasov-Poisson equation. ('A paraître).

[23] R. Robert \& C. Rosier, On the modelling of small scales for $2 \mathrm{D}$ turbulent flows, $J$. Stat. Phys. 86, 3/4, 1997.

[24] R. Robert \& C. Rosier, A propos de l'effet papillon, (À paraître).

[25] R. Robert \& J. Sommeria, Statistical equilibrium states for two-dimensional flows, J. Fluid Mech. 229, (1991), 291-310.

[26] R. Robert \& J. Sommeria, Relaxation towards a statistical equilibrium state in two-dimensional perfect fluid dynamics, Phys. Rev. Letters 69, (1992), 2276-2279.

[27] J. Sommeria, C. Staquet \& R. Robert, Final equilibrium state of a two-dimensional shear layer, J. Fluid Mech. 233, (1991), 661-689.

[28] A. Thess, J. Sommeria \& B. Jüttner, Inertial organization of a two-dimensional turbulent vortex street, Phys. Fluids 6(7), (1994), 2417-2429.

[29] V.I. Youdovitch, Non-stationary flow of an incompressible liquid, Zh. Vych. Mat. 3, (1963), 1032-1066.

[30] L.C. Young, Generalized surfaces in the calculus of variations, Ann. Math. 43, (1942), 84-103. 